\title{
Conceptual and Experimental Results of the Transverse Normal Zone Propagation in the B0 ATLAS-Barrel Model Coil
}

\author{
François-Paul Juster, Alexey V. Dudarev, Philippe Fazilleau, and François Kircher
}

\begin{abstract}
The normal zone propagation velocity is an important feature in the design of a superconducting magnet. In the particular case of the ATLAS-Barrel toroid coil protection, it is all the more important as the fast discharge is essentially realized through the toroid windings by exciting quench heaters. The time constant of that fast discharge, and consequently the final magnet temperature, are therefore determined by the heaters activation time constant and the quench propagation kinetics. Taking into account the particular Race-track shape of the ATLAS-Barrel coil, the transverse quench propagation dominates the duration of the fast discharge.

In this paper, we present, at first, the experimental measurements of the transverse normal zone propagation velocities made at CERN in 2002 on the B0 model-coil up to a maximal $24 \mathrm{kA}$ operating current. Then, we present a code developed in our laboratory, based upon CAST3M finite-element code, which allows the computation, among others, of the transverse normal zone propagation velocities. We finally compare computation and experimental results and obtain a very satisfactory agreement in a large (15-24 kA) operating current range.
\end{abstract}

Index Terms-Current diffusion, finite-element computation, magnet protection, normal zone propagation.

\section{INTRODUCTION}

$\mathbf{T}$ HE B0 coil has been successfully tested at CERN in 2002. Let us briefly remind that $\mathrm{B} 0$ is a mock-up of one out of the 8 racetrack-shaped coils that form the ATLAS Barrel Toroid and mainly differs from one of these coils by the length reduced from $26 \mathrm{~m}$ to $9 \mathrm{~m}$. B0 is composed of two parallel double-pancakes. Each double-pancake includes two layers of 30 conductors. B0 main characteristics are summarized in Table I. It must be noted that an insulated thermal drain (aluminum RRR 25) has been set on one side of the double-pancakes (Fig. 1).

An extensive tests campaign has been carried out to measure the thermal, mechanical and electromagnetic properties of the coil [1]. We focus here on the protection of the magnet. Rather than a fast discharge in a dedicated external dump resistor, it has been chosen, in case of fault scenario, to dissipate most of the magnetic energy of the coils through the windings and the coils casing [2]. This is done by means of resistive heaters which initiate quenches in each coil. The final hot spot tem-

Manuscript received October 20, 2003.

F.-P. Juster, P. Fazilleau, and F. Kircher are with CEA Saclay, DSM/DAPNIA/ SACM 91191 Gif Sur Yvette, France (e-mail: juster@dapnia.Cea.fr; fazilleau@dapnia.cea.fr).

A. V. Dudarev is with CERN, Geneva 23, CH-1211, Switzerland. Digital Object Identifier 10.1109/TASC.2004.830567
TABLE I

$\mathrm{B}_{0}$ MAIN CHARACTERISTICS

\begin{tabular}{llll}
\hline \hline Sc Cable type & Rutherford & Operating Current & $20500 \mathrm{~A}$ \\
Strand Number & 40 & Peak Field & $3.6 \mathrm{~T}$ \\
Cu/Sc Ratio & 1.19 & Turns/layer & 30 \\
Strand Diameter & $1.3 \mathrm{~mm}$ & Inter layer Insul. & $0.4 \mathrm{~mm}$ \\
Stabilizer & Aluminum & Ground plane Insul. & $1.0 \mathrm{~mm}$ \\
$\mathrm{I}_{\mathrm{c}} @ 4.2 \mathrm{~K}, 5 \mathrm{~T}$ & $66 \mathrm{kA}$ & Cooling Mode & Indirect \\
RRR Al @ 0 T & 1100 & 2-ph helium temp. & $4.5 \mathrm{~K}$ \\
Bare Conductor & $57312 \mathrm{~mm}^{2}$ & Inductance & $0.22 \mathrm{H}$ \\
Cond. Insulation & $0.4 \mathrm{~mm}$ & Stored energy & $46 \mathrm{MJ}$ \\
\hline \hline
\end{tabular}

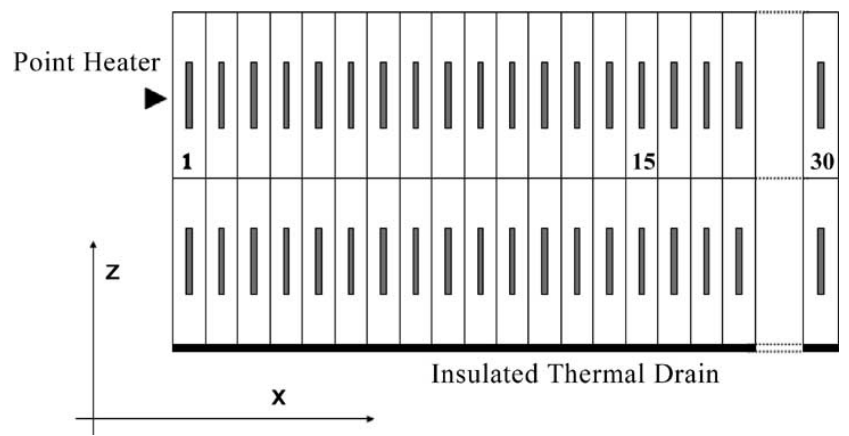

Fig. 1. B0 coil. Double-pancake cross-section.

perature of the winding is determined by the magnet discharge time constant and therefore by the evolution of its resistance. It is consequently of outstanding importance to know the quench propagation kinetics inside the windings.

Taking into account the racetrack shape of the coils $(26 \mathrm{~m}$ $35 \mathrm{~m}$ ), the development of the normal zone is dominated by the transverse turn-to-turn propagation velocity along the $\mathrm{x}$-axis (Fig. 1): if the longitudinal propagation were the only permitted normal zone propagation mode and assuming a maximum $10 \mathrm{~m} / \mathrm{s}$ propagation velocity at nominal current [3], 1 to 2 minutes would be necessary to quench the whole magnet. Thanks to the transverse mode, this time is reduced to a few seconds [3]. That leads us to turn our intention in this paper on the transverse normal zone propagation velocity and its dependence with the magnet current.

In a first part, we shall present the experimental results, and, in a second part their comparison with the values predicted by a code developed in our laboratory, based on a 2-d finite-element method. 


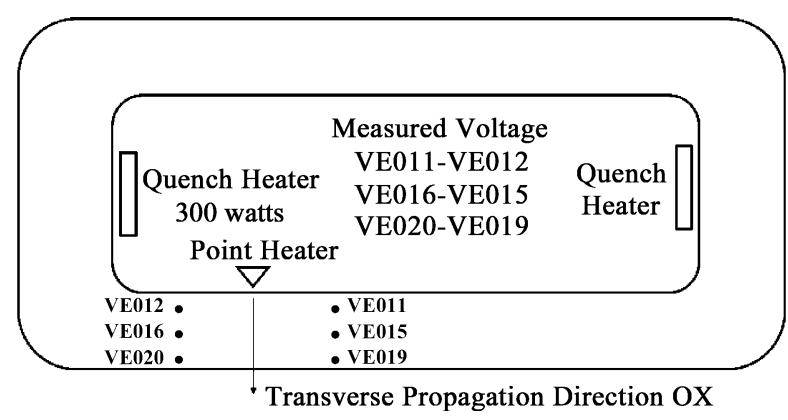

Fig. 2. Sketch of B0 coil. VE011-VE012 is the voltage measured on the inner conductor $\left(\mathrm{N}^{\circ} 1\right)$ of the external layer of the double-pancake, VE016-VE015 is measured on the middle conductor $\left(\mathrm{N}^{\circ} 15\right)$ and VE020-VE019 on the outer one $\left(\mathrm{N}^{\circ} 30\right)$. Only one double-pancake is figured.

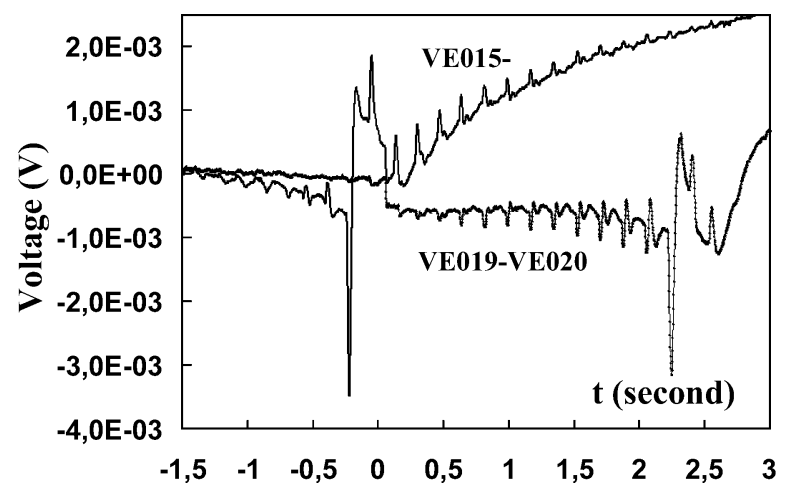

Fig. 3. Voltage taps measurements. Time steps between successive transitions. $\mathrm{I}=21 \mathrm{kA}$. Quench initiated by E013A point heater.

\section{MEASUREMENTS}

\section{A. Introduction}

The transitions at various magnet currents can be induced by two kinds of heaters acting on the inner part of the conductors layers (Conductor $\mathrm{N}^{\circ} 1$, Fig. 1):

1) The above-mentioned protection quench heaters which are resistive-type. Those quench heaters ( $300 \mathrm{~W}$ ) typically deposit a few hundreds joules in both layers of a double-pancake. There are 2 symmetrically disposed quench heaters on each double-pancake centered on the small straight parts of the racetrack.

2) The 'Point Heaters' which are capacitive-type. They typically deposit a 1 to 10 joules amount of energy locally in the external layer of a double-pancake. They are located at the entrance of the long straight part of the racetrack. Let us also mention that point heaters have also been placed on the outer part of the layer (Conductor $\mathrm{N}^{\circ} 30$ ), but were used only one time.

The transverse propagation velocities measurements are made by means of voltage taps located in the vicinity of the point heaters. Three couples of taps $(0.8 \mathrm{~m}$ apart $)$ are placed at the entrance of the coil long straight part near the point heaters. As it may be seen on Fig. 3, voltage taps act as pick-up coils and allow the determination of the time steps between successive transitions of adjacent conductors.

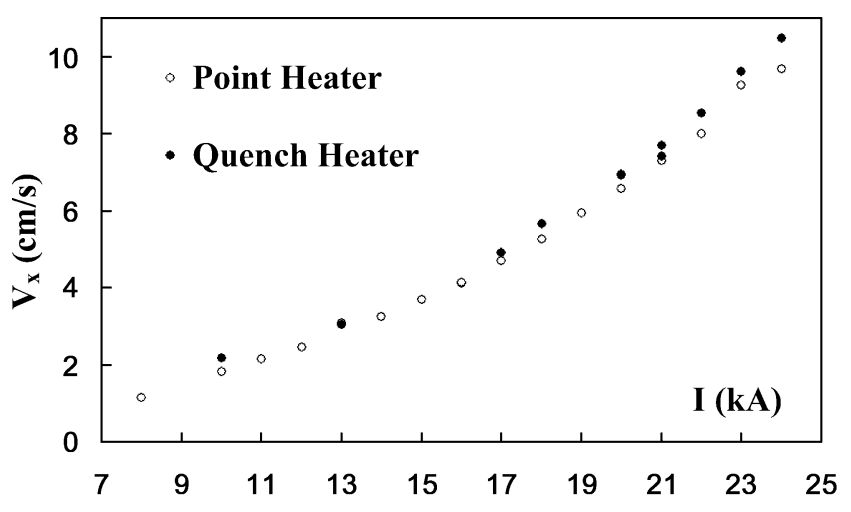

Fig. 4. Transverse (x-axis) normal zone propagation velocities versus magnet nominal current.

\section{B. Experimental Results}

To present the experimental results, we chose to compute the average value of the measured time steps between the middle conductor $\left(\mathrm{N}^{\circ} 15\right)$ and the outer conductor $\left(\mathrm{N}^{\circ} 30\right)$. It must be noticed that these time steps are not constant and exhibit a typical 63 to 5\% dispersion depending on the current. We considered that the indications given by the innermost conductor transitions were 'contaminated' by a preheating effect due to the closeness of the heaters and as a consequence do not lead to pure propagation velocities determination.

The results are presented on Fig. 4 in the classical form of velocity expressed in length by unit of time. We considered a $12.8 \mathrm{~mm}$ overall conductor dimension along the $\mathrm{x}$-axis.

Most of the measurements were made with point heater quench ignition. From 8 to $24 \mathrm{kA}$, the here-above defined average time step had been found to vary from $120 \mathrm{~ms}$ to $1.1 \mathrm{~s}$ which correspond, to respectively 10.7 and $1.2 \mathrm{~cm} / \mathrm{s}$.

\section{Comments}

We remark that the measurements made with quench heater ignition systematically (with only one exception) lead to higher velocities compared to those made with point heater ignition. We can put forward two reasons to this:

1) An excessive preheating due to the quench heater.

2) The normal zone size evolution, others things being equal, is driven by the magnetic field: the quench propagation velocities are high in high field region and, inversely, low in low field regions. When activated by the quench heater, the normal zone is created in a region where the magnetic field is higher than in the vicinity of the point heater where the measurements are made. As a consequence, the normal zone propagation front tends to slow down when approaching the measurements zone. We think that this slowing-down effect requires some delay and therefore the effective measured velocity is higher than the velocity really corresponding to the local field, velocity which is more properly measured with point heater ignition. 
TABLE II

INSULATION THERMAL PROPERTIES

\begin{tabular}{ccc}
\hline \hline $\begin{array}{c}\text { Temperature } \\
(\mathrm{K})\end{array}$ & $\begin{array}{c}\text { Thermal Conductivity } \\
(\mathrm{W} \cdot \mathrm{m} / \mathrm{K})\end{array}$ & $\begin{array}{c}\text { Specific Heat } \\
\left(\mathrm{J} / \mathrm{m}^{3} \cdot \mathrm{K}\right)\end{array}$ \\
\hline 4 & 0.060 & 3927 \\
6 & 0.084 & 10642 \\
8 & 0.100 & 19536 \\
10 & 0.114 & 29913 \\
\hline \hline
\end{tabular}

\section{COMPUTATIONS}

\section{A. Assumptions}

Our code is based on the finite-element code CAST3M [4] and numerically solves the heat equation in transient regime. We introduced personal procedures to treat particular problems of superconducting magnets: the minimum quench energy and the normal zone propagation.

1) Geometrical Assumptions: All the computations are made in 2-d. There are no structural obstacles in 3-d modeling other than the excessively long computation times. A complete B0 cross-section is modeled i.e., 2 double-pancakes (120 insulated conductors) with their inter-layer and ground plane insulation, in their coil casing. A particular attention has been attached to a fine meshing of all the insulating material. The 4-tube cooling system is also modeled with adjustable heat-exchange coefficient. The insulated thermal drain is included.

2) Magnetic Assumptions: The magnetic field variation all-over the cross-section is taken into account. The field, including the magnetic mirror contribution [1], has been computed in the measurement cross-section. At $20.5 \mathrm{kA}$, the field varies from 1.5 tesla (inner layer, Cond. $\mathrm{N}^{\circ} 20$ ) to 3.5 tesla (outer layer, Cond. $\mathrm{N}^{\circ} 1$ ). In our code, it means that each of the 120 conductors have their own current-sharing and critical temperatures.

3) Thermal Properties: The dependence with temperature of thermal properties of each material is taken into account. The computations are dominated by the electrical resistivity of the aluminum and the thermal properties of the conductor insulation.

For the Aluminum, we assume an identical RRR value for all the conductors. This value is computed from the magneto resistance measurements [5], [6], considering the average field in a cross-section (above 1 tesla, the RRR value does not vary significantly). We consider a $440 \mathrm{RRR}$ value at $15 \mathrm{kA}$ and a constant 400 RRR value above $20 \mathrm{kA}$. Concerning the insulation, we considered the properties of G10 resin-epoxy [7], [8]. They are summarized in Table II.

4) Boundary Conditions: During the tests, coil winding temperatures comprised between 4.5 and $4.9 \mathrm{~K}$ have been measured. We therefore carried out 2 sets of computations with initial temperatures of $4.5 \mathrm{~K}$ or $4.9 \mathrm{~K}$ imposed all over the cross-section, except for the conductor $\mathrm{N}^{\circ} 1$ of the outer layer where a temperature step is imposed. Afterwards, the normal zone evolution is computed at current varying from 15 to $24 \mathrm{kA}$.

5) Deposited Power: In a large Al-stabilized conductor, in case of transition the current does not redistribute instanta-

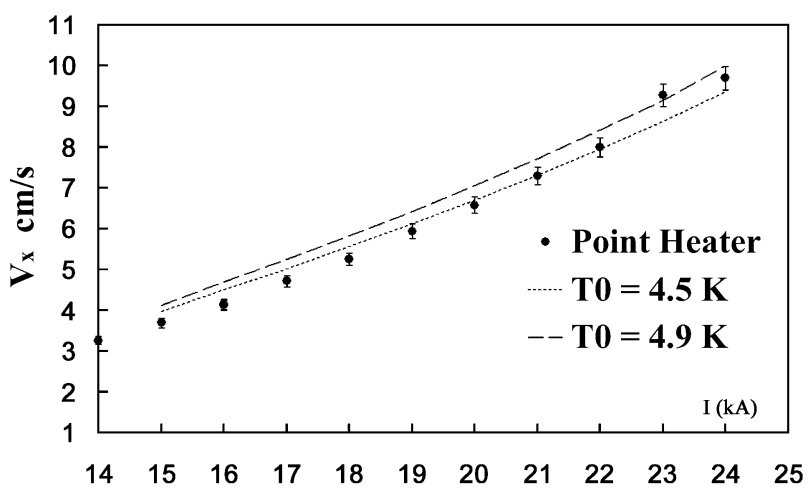

Fig. 5. Comparison between computed and measured transverse normal zone propagation velocities versus magnet nominal current. Point heater measurements.

neously in the aluminum cross-section. This phenomenon is governed by the equation:

$\Delta \mathrm{j}=\mathrm{D}_{\mathrm{m}} \cdot \frac{\angle \mathrm{j}}{\angle \mathrm{t}}$ with $\mathrm{D}_{\mathrm{m}}=\frac{\rho}{\mu_{0}}, \rho$ is the electrical resistivity.

During this 'current diffusion' process (timeconstant $\approx 1 \mathrm{~s}$ ) [9], [10], an overheating occurs. The here-above equation is numerically solved as well by 2-D finite-element method in the conductor cross-section, independently of the main thermal program. The current density variation $j(x, z, t)$, and thus the generated heat $\rho j^{2}$, versus space and time are computed. Then, the $\rho j^{2}$ term is averaged in the conductor cross section, is introduced in the main thermal program and is affected to a particular conductor as soon as it reaches its own current-sharing temperature.

The current-sharing line, which corresponds to a progressive expulsion of the current from the superconductor, is included in the current-diffusion computation as a progressive variation of the resistivity of the superconductor [11]. As the temperature field evolution, necessary to build this equivalent variation, is not a priori known, iterations between thermal and current-diffusion computations are required.

\section{B. Computation Results}

Considering the remarks made in II-C-2 and the assumed boundary conditions, the computations are supposed to reproduce point heater ignition measurements. The direct comparison of those measurements and the computations results is illustrated in Fig. 5. The plotted computed values are, as for the measurements, the average velocities between conductors $\mathrm{N}^{\circ} 15$ and 30.

First of all, one may state a very satisfactory agreement, for such kind of computation, between experimental and calculated values: a few percent at high current and $10 \%$ at low current. It must be noted, that a $10 \%$ error on transverse Ox velocity determination leads to a 0.2 to $0.3 \mathrm{~K}$ error on the final hot spot temperature $T_{\text {hs }}$ after a quench at nominal current $(\mathrm{I}=20.5 \mathrm{kA}$, $\mathrm{T}_{\mathrm{hs}} \approx 81 \mathrm{~K}$, assuming a longitudinal propagation velocity $\mathrm{V}_{1}=$ $10 \mathrm{~m} / \mathrm{s})$ and $0.1 \mathrm{~K}$ at $16 \mathrm{kA}\left(\mathrm{T}_{\mathrm{hs}} \approx 64 \mathrm{~K}, \mathrm{~V}_{\mathrm{l}}=3 \mathrm{~m} / \mathrm{s}\right)$. The accuracy of the computations is thus sufficient for the magnet protection design. 


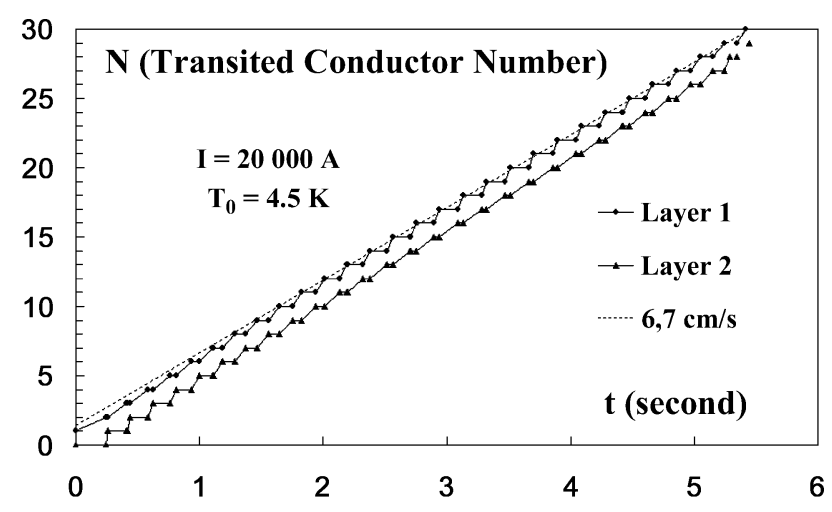

Fig. 6. Evolution of the transited conductors number on each layer of the heated double-pancake versus time. Dashed line slope indicates the $6.7 \mathrm{~cm} / \mathrm{s}$ average velocity computed between conductors number 15 and 30 .

\section{Discussion}

1) A Typical Computation Result: Fig. 6 shows the evolution of the transition of the two layers of the heated double-pancake at $20 \mathrm{kA}$. A slight $\pm 2 \%$ variation of the velocity has been computed between conductors $\mathrm{N}^{\circ} 15$ and 30 . This effect is lower at low currents $( \pm 0.5 \%$ at $15 \mathrm{kA})$ and higher at high current $( \pm 3.5 \%$ at $24 \mathrm{kA})$. Those values are similar to the observed experimentally velocity variations (plotted as error bars on Fig. 5). One shall note that the computed velocities are quasi equal in the two layers in spite of the magnetic field difference: the transverse ( $\mathrm{z}$-axis in Fig. 1) thermal coupling between layer leads actually to the prevalence of the layer submitted to the highest field i.e., where the intrinsic velocity is higher.

2) Helium Cooling: In the 15-24 kA current range studied, no significant effect of the helium cooling on transverse velocity has been found ( $<1 \%$ at $15 \mathrm{kA}$ ) even with $10000 \mathrm{~W} / \mathrm{m}^{2} \mathrm{~K}$ helium convection heat-exchange coefficient. Practically, the effect of high coefficient is damped by the high thermal resistance due to the gluing of the cooling tubes on the casing.

3) Thermal Drains: The advantage of thermal drains has been widely discussed all along the project. The computations show that the transverse propagation is not significantly enhanced by the 1-mm-thick thermal drains: $3 \%$ at $16 \mathrm{kA}, 2 \%$ at $21 \mathrm{kA}$ and $1 \%$ at $24 \mathrm{kA}$. A $10 \%$ improvement at $20.5 \mathrm{kA}$, would require 400 RRR aluminum drains. Such purity is very hard to achieve under laminated form.

4) Limits of the Model: We nevertheless observe a global increasing deviation between experimental and computations results as the magnet current decreases. This is interpreted as a 3-D effect: the 2-D assumption is rigorously valid only if the temperature field does not depend on the longitudinal coordinate (infinite longitudinal propagation velocity). We think that the pertinent characteristics that must be compared to evaluate the limits of the model are the two following characteristic times:

Longitudinal propagation process: $\mathrm{t}_{\mathrm{l}} \approx \mathrm{D}_{\mathrm{t}} / \mathrm{v}_{1}^{2}\left(\mathrm{D}_{\mathrm{t}}\right.$ : thermal diffusivity of Aluminum $\approx 2 \mathrm{~m}^{2} / \mathrm{s}, \mathrm{v}_{1}$ longi- tudinal propagation velocity), it represents the order of magnitude of the time required to lead an initially cold point of the magnet to normal resistive state when it is reached by the propagation front [10].

- Transverse propagation process: $t_{t}=$ time step between transitions of adjacent conductors.

At high current (20-24 kA) [3], [12], $\eta=\mathrm{t}_{\mathrm{l}} / \mathrm{t}_{\mathrm{t}} \approx 10^{-2}-$ $10^{-1}$, the ratio is small, indicating a weak coupling between the transverse and longitudinal modes, the model is therefore applicable. This ratio quickly increases as current decreases:

$\eta \approx 0.3$ at $15 \mathrm{kA}$, which we considered as the low limit of our 2 -D model validity. At $10 \mathrm{kA}, \eta \approx 3$ : a full 3 -D model should probably be required.

\section{CONCLUSION}

The experimental and 2-d finite-elements computations results of transverse normal zone propagation velocities carried out on B0 coil are in a satisfying $0-10 \%$ agreement in the 15-24 kA current range. This precision is largely acceptable for protection design purposes, dominated here by the transverse turn-to-turn propagation mode. The next step is now to fill up our computations development in validating our model on the longitudinal propagation velocity measurements.

\section{ACKNOWLEDGMENT}

The authors would like to thank all the members of the ATLAS magnet team in CEA, CERN and INFN/LASA, who successfully carried out the tests campaign on B0 coil.

\section{REFERENCES}

[1] P. Miele et al., "ATLAS B0 toroid model coil test at CERN," IEEE Trans. Apll. Supercond., vol. 12, no. 1, pp. 411-415, March 2002.

[2] “ATLAS Barrel Toroid Technical Design Report,", CERN/LHC/97-19 ATLAS TDR 7, April 30, 1997.

[3] A. V. Dudarev et al., Quench Evolution and Hot Spot Temperature in the ATLAS B0 Model Coil, this conference.

[4] http://www-cast3m.cea.fr/ [Online]

[5] C. Berriaud, "ATLAS Reports,", 5 M 2900 T-2 000027 98, May 1998.

[6] —-, "ATLAS Reports,", 5 M 2900 T-2 000037 98, January 1999.

[7] M. B. Kasen et al., "Mechanical, electrical and thermal characterization of G-10CR and G-11CR glass-cloth/epoxy laminates between room temperature and $4 \mathrm{~K}$," in Advance in Cryogenics Engineering (Material): Plenum Press, vol. 26, pp. 235-244.

[8] http://cryogenics.nist.gov/ [Online]

[9] M. N. Wilson, Superconducting Magnets: Clarendon Press Oxford.

[10] Y. Iwasa A, Case Studies in Superconducting Magnets, Design and Operational Issues: Plenum Press, 1994.

[11] Y. Lvovsky, "Normal zone propagation model for monolith conductors with current diffusion," IEEE Trans. Appl. Supercond., vol. 13, no. 2, pp. 1688-1691, June 2003.

[12] E. W. Boxman et al., "Current diffusion and normal zone propagation inside the aluminum stabilized superconductor of ATLAS model coil," IEEE Trans. Appl. Supercond., vol. 13, no. 2, pp. 1684-1687, June 2003.

[13] F. P. Juster et al., "Stability and quench propagation velocities measurements on the 'race-track' mockup of ATLAS toroid coil," IEEE Trans. Appl. Supercond., vol. 10, no. 1, pp. 677-680, March 2000. 